\title{
Automodulação espacial de fase: uma segunda demonstração instrutiva e de baixo custo de um efeito óptico não linear
}

Spatial self-phase modulation: a second instructive and low-cost demonstration of a nonlinear optical effect

\author{
Marlon S. Ribeiro ${ }^{1}$, Gerson K. da Cruz $^{1}$, Sergio L. Gómez ${ }^{*} @$, Rozane F. Turchiello² \\ ${ }^{1}$ Universidade Estadual de Ponta Grossa, Departamento de Física, Ponta Grossa, PR, Brasil \\ ${ }^{2}$ Universidade Tecnológica Federal do Paraná, Departamento de Física, Ponta Grossa, PR, Brasil
}

Recebido em 12 de Julho, 2018. Revisado em 20 de Julho, 2018. Aceito em 22 de Julho, 2018.

\begin{abstract}
No ensino tradicional da Física Geral, o ensino da óptica normalmente se restringe à denominada óptica linear, negligenciando a óptica não linear, a qual desempenha um papel cada vez mais importante em diversas áreas do desenvolvimento tecnológico. A presente comunicação aborda a proposta de um experimento simples e de baixo custo para a demonstração do efeito óptico não linear denominado automodulação espacial de fase. Este experimento vem complementar a proposta de experimentos de baixo custo na demonstração de efeitos ópticos não lineares que começou com a demonstração da chamada lente térmica num artigo anterior. A automodulação espacial de fase baseia-se na introdução de um termo adicional na fase da onda gaussiana incidente num meio como conseqüência de seu aquecimento pelo feixe. Como meio não linear é proposta a utilização de um fluido de simples obtenção e baixo custo, como molho a base de soja (molho shoyu), suco de uva integral ou um refrigerante cola.
\end{abstract}

Palavras-chave: óptica não linear, automodulação espacial de fase, feixe gaussiano, interferência.

The traditional teaching of general physics at undergraduate level usually restricts to linear optics, neglecting the nonlinear optics which plays an increasingly important role in several areas of technological development. This paper discusses the proposal of a simple and low cost experiment for the demonstration of the nonlinear optical effect called spatial self-phase modulation. This experiment completes the proposal of low cost experiments in the demonstration of nonlinear optical effects that began with the demonstration of the so-called thermal lens in a previous paper. Spatial self-phase modulation is based on the introduction of an additional term in the phase of a Gaussian wave incident on a medium as a consequence of its heating by the beam. As a nonlinear medium it is proposed to use a simple and low cost fluid such as soy sauce (shoyu sauce), whole grape juice or cola soft drink. Keywords: nonlinear optics, spatial self-phase modulation, gaussian beam, interference.

\section{Introdução}

A grade curricular das disciplinas de física geral nos cursos de ciências básicas e engenharias geralmente incluem os aspectos básicos da denominada óptica geométrica e da óptica física, relegando um estudo mais aprofundado de temas importantes em ambas áreas da óptica ao campo de disciplinas optativas, quando forem elas ofertadas. O ensino da denominada óptica não linear [1, a qual se baseia nos efeitos da interação de um feixe de luz com um meio material, atuando na modificação da propagação do feixe original quanto na geração de novas ondas eletromagnéticas, não deveria ser visto como uma simples atualização de conhecimento acadêmico com o intuito de manter atualizada a comunidade, quando em geral existe um tempo limitado para a aboradagem pedagógica das disciplinas que formarão o futuro cientista. É inegável que uma grande parcela do estágio atual do

*Endereço de correspondência: sgomez@uepg.br. desenvolvimento científico e tecnológico descansa sobre os avanços na área da óptica não linear. Efeitos ópticos não lineares, vistos na época do descobrimento do laser como problemas, dão origem a aplicações tecnológicas que vão da área das comunicações à medicina. Assim, é fundamental um conhecimento básico desses fenômenos para uma correta contextualização do ensino da óptica. Num artigo prévio 2 propusemos a utilização do denominado efeito de lente térmica (thermal lensing) para introduzir o ensino da óptica não linear num curso de física geral ou como um projeto de iniciação científica. Neste artigo propomos a utilização de um segundo fenômeno óptico não linear que é possível de ser observado sem a necessidade de um complexo aparato experimental ou amostras de dificil preparação. O fenômeno proposto é o denominado automodulação espacial de fase (spatial self-phase modulation) de um feixe gaussiano incidente sobre uma amostra que apresente alta absorção óptica no comprimento de onda do feixe, o qual leva à formação de 
um padrão de interferência formado por um conjunto de anéis no campo afastado. Como no caso da lente térmica, este efeito é produzido pela incidência sobre um meio de um feixe com um perfil espacial de intensidade representado por uma função gaussiana. Se o meio absorve a luz, uma parte da energia eletromagnética do feixe é devolvida, após a relaxação, na forma de calor, aquecendo o meio. O índice de refração $(n)$ de um material em geral é função do comprimento de onda da luz $(\lambda)$ e da temperatura $(T)$, entre outros. Assim, o aquecimento do meio seguindo um padrão espacial específico leva a uma mudança correlata do índice de refração. A automodulção espacial de fase tem sido usada para obter, por exemplo, o rendimento quântico de fluorescência de moléculas em solução 3] e o coeficiente termo-óptico de líquidos 4]. Na Seção II faremos uma apresentação dos fundamentos da automodulação espacial de fase e na Seção III apresentaremos uma montagem experimental simples para a observação da formação da automodulação espacial utilizando como amostra um molho de uso comercial.

\section{Fundamentação teórica}

\subsection{Feixe Gaussiano}

Os lasers de uso em pesquisa em geral produzem um feixe de luz laser que possui uma distribuição espacial de intensidade que é representada por uma função gaussiana a qual é dada por [5]:

$$
I(r)=I_{o} e^{-2 \frac{r^{2}}{w^{2}}}
$$

onde $I_{o}$ é a intensidade no centro do feixe $(r=0)$ e $w$ é o parâmetro denominado raio do feixe gaussiano. Este valor é determinado pela distância ao centro do feixe onde a intensidade diminui para o valor $\frac{I_{o}}{e^{2}}$ e pode ser medido pelo procedimento apresentado na Ref. 6]. Para um feixe gaussiano que se propaga ao longo do eixo $z$ e é focalizado por uma lente convergente, o valor do raio do feixe gaussiano e do raio de curvatura da frente de onda $(R)$ à distância $z$ do foco da lente são dados, respectivamente, por:

$$
w(z)^{2}=w_{o}^{2}\left[1+\left(\frac{\lambda z}{2 \pi w_{o}^{2}}\right)^{2}\right]
$$

e

$$
R(z)=z\left[1+\left(\frac{2 \pi w_{o}^{2}}{\lambda z}\right)^{2}\right]
$$

onde $w_{o}$ é o raio do feixe na posição do foco da lente e $\lambda$ é o comprimento de onda da luz laser. Como mostra a Eq. 3, o raio de curvatura na posição do foco tende ao infinito, ou seja a frente de onda é plana. A Fig. 1 apresenta uma representação 3D de um feixe gaussiano.

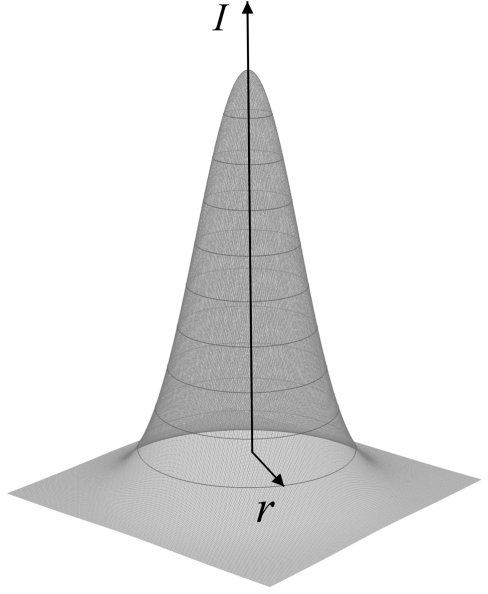

Figura 1: Representação 3D da distribuição espacial de intensidade de um feixe gaussiano. $r$ é a distância radial ao eixo $z$ (direção de propagação do feixe).

\subsection{Automodulação espacial de fase}

O índice de refração de um material depende, entre outros fatores, da temperatura do meio, podendo ser escrito como:

$$
n(T+\triangle T)=n_{\mathrm{o}}(T)+\frac{d n}{d T} \triangle T,
$$

onde $n_{\mathrm{o}}(T)$ é o índice de refração linear correspondente à temperatura $T$, a qual pode ser a temperatura ambiente, $\frac{d n}{d T}$ é o denominado coeficiente termoóptico e $\triangle T$ representa a variação de temperatura do meio em relação à temperatura $T$. No caso de um meio que possui absorção óptica linear $\alpha(\lambda)$, parte da energia eletromagnética do feixe é transformada em calor, produzindo portanto o aquecimento do meio. Em primeira aproximação podemos considerar que o aquecimento experimentado pelo meio é proporcional à intensidade da luz incidente sobre ele e ao coeficiente de absorção $(\triangle T \propto \alpha I)$.

Ou seja, no caso de um meio absorvedor, a absorção da luz com o perfil radial descrito pela Eq. 1] leva a um aquecimento não uniforme do meio mas com simetria radial, ou seja $\triangle T=\triangle T(r)$. Assim, o índice de refração do meio iluminado por um feixe laser gaussiano, terá um perfil espacial também gaussiano. A mudança do índice de refração leva por sua vez a uma mudança adicional da fase da onda a qual é dada por

$$
\varphi_{N L}(r)=l \frac{2 \pi}{\lambda} \frac{d n}{d T} \triangle T(r) \propto \alpha I(r) \propto \alpha P e^{-2 \frac{r^{2}}{\omega^{2}}},
$$

onde $l$ é a espessura da amostra e $P$ é a potência do laser. No ponto focal da lente convergente, a frente de onda do feixe laser é representado por uma onda plana $(R \rightarrow \infty)$ e portanto o vetor de onda $\mathbf{k}$ é paralelo à direção de propagação do feixe $(z)$. Por outro lado e como resultado do aquecimento do meio, no plano de saída da amostra e a uma distância $r$ do centro do feixe, a fase da onda será acrescida do valor $\varphi_{N L}(r)$. A equação básica da óptica geométrica é dada pela equação eikonal $\nabla S=n \hat{s}$, 
onde $S$ é a função escalar conhecida como eikonal e $\hat{s}$ é o vetor unitário tangente à trajetoria do raio (direção de propagação do feixe) e normal à frente de onda, a qual é dada por $S(\mathbf{r})=$ cte $[7$. O ganho adicional na fase da onda na saída da amostra implica portanto que o vetor de onda terá uma componente adicional a qual é radial e dada por:

$$
k_{\perp} \propto \nabla_{r} \varphi_{N L}(r) \propto r e^{-2 \frac{r^{2}}{\omega^{2}}}
$$

As Figuras 2a e 2b mostram gráficos da função gaussiana e de sua derivada, respectivamente. Como é possível ver nessas figuras, a derivada da função gaussiana apresenta dois valores similares a ambos os lados do ponto de inflexão da gaussiana. Assim, desses dois pontos partirão respectivos raios os quais se propagarão na mesma direção $\mathbf{k}=\mathbf{k}_{z}+\mathbf{k}_{\perp}$, interferindo no campo afastado (Difração de Fraunhofer) [5]. Um cálculo mais preciso do padrão de difração gerado pela automodulação de fase pode ser obtido mediante o cálculo da integral de difração 8 .

\section{Montagem experimental}

Para a observação dos anéis de interferência como resultado do efeito de automodulação espacial utilizaremos
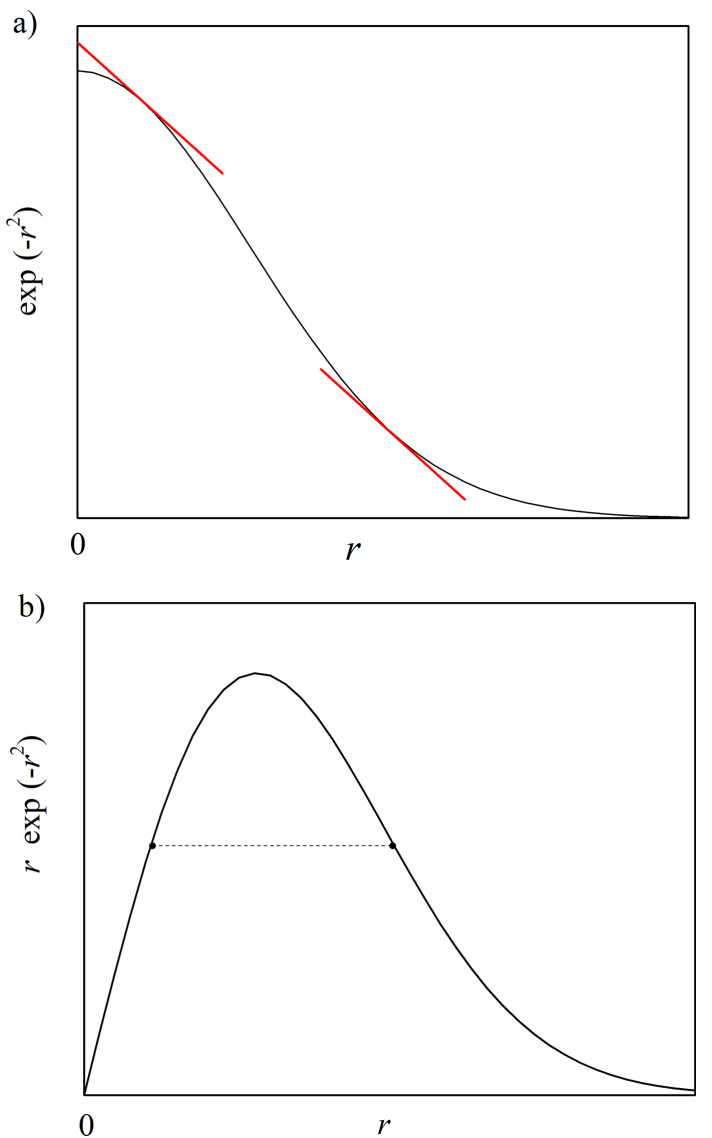

Figura 2: Gráficos da a) função gaussiana e b) derivada da função gaussiana. As linhas vermelhas são tangentes em duas posições, ao redor do ponto de inflexão da função gaussiana, que possuem a mesma derivada. como meio absorvedor um fluido de fácil obtenção: molho shoyu, um molho a base de soja, e como material de controle será utilizada água pura. O molho shoyu comercial é um fluido de cor escura intensa, ou seja, possui uma grande absorção da luz visível. Para fins didáticos, qualquer marca de molho shoyu pode ser utilizado. Uma lista não definitiva de fluidos que podem ser utilizados com bons resultados, como pudemos conferir, inclui o suco integral de uva e o refrigerante cola. A amostra pode ser acondicionada num porta-amostra como descrito na Ref. 2]. Outros porta-amostras que podem ser utilizados são cubetas de vidro usadas em espectroscopia. Em geral para amostras com grande absorção óptica recomenda-se o uso de porta-amostras finos.

A montagem experimental para a observação da automodulação espacial do feixe gaussiano emprega os seguintes elementos:

- Laser com feixe gaussiano

- Lente convergente

- Amostra

- Anteparo

A Fig. 3 apresenta uma representação esquemática da montagem experimental para a observação da automodulação espacial de fase. A lente convergente é usada para aumentar a intensidade do feixe, o qual acontece ao concentrar a energia luminosa numa área menor, facilitando a observação do efeito óptico não linear se o laser não for muito intenso. Em relação à distância focal da lente convergente, quanto menor for, melhor é a visualização dos anéis de interferência.

Se o laser não tiver controle da potência, é possível controlá-la mediante um polarizador se o laser for linearmente polarizado ou um conjunto de dois polarizadores lineares no caso de um laser não polarizado, posicionados em seqüência entre a saída do laser e a lente, tirando proveito da lei de Malus [5].

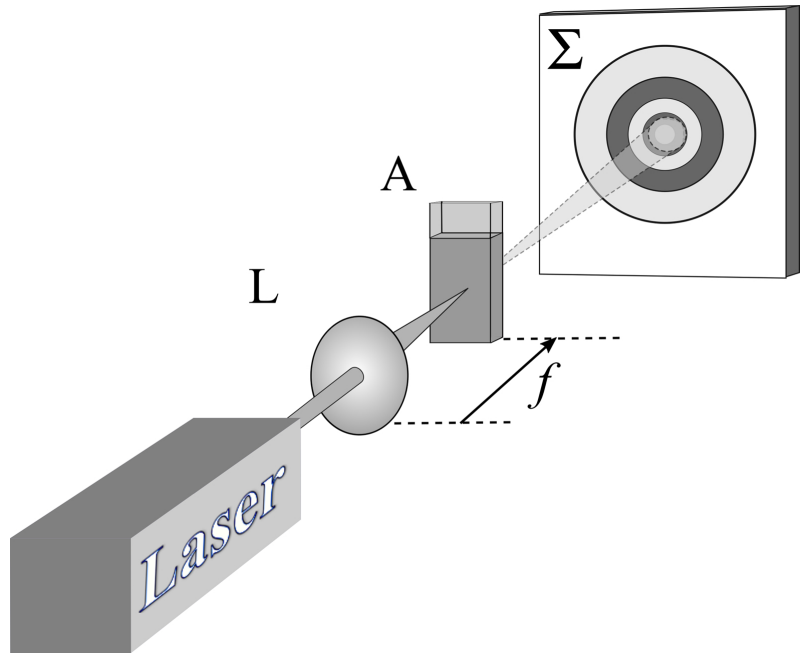

Figura 3: Arranjo experimental: lente (L) de distância focal $f$, porta-amostra $(\mathrm{A})$ e anteparo $(\Sigma)$. 
O porta-amostra deve ser posicionado no foco da lente convergente e a razão para isto é a seguinte: a óptica geométrica ensina que um raio passando pelo centro de uma lente delgada não tem o seu percurso modificado. Assim, mesmo que o feixe de laser induza uma lente térmica no meio, esta não levará a uma mudança visível no tamanho da imagem do feixe sobre o anteparo no campo afastado em comparação a quando não está a amostra presente, como seria no caso da amostra estar a uma certa distância do foco 2 .

\section{Procedimento Experimental}

No arranjo posicionamos o porta-amostra contendo água pura na posição do foco da lente e posicionamos o anteparo a uma distância de aproximadamente $2 \mathrm{~m}$ da lente (pode-se também projetar diretamente a imagem sobre a parede, já que o diâmetro dos anéis formados podem ser da ordem de $50 \mathrm{~cm}$ ). Começando com uma potência baixa, aumentamos a mesma gradualmente. Poderemos comprovar que para todas as potências a imagem observada sobre o anteparo, um círculo luminoso, não muda de tamanho mas se torna cada vez mais intenso. Assim, comprovamos a ausência da automodulação espacial de fase pela ausência dos anéis de interferência. O coeficiente de absorção óptica da água na região visível do espectro eletromagnético é desprezível. Na sequência, colocamos o porta-amostra com molho shoyu ou algum dos outros fluidos sugeridos no foco da lente convergente e é escolhida a menor potência possível do laser. Para esse valor provavelmente não haverá a formação de anéis, observando-se somente a imagem do feixe como o obtido com a amostra de água. Na sequência, aumentamos a potência incidente sobre a amostra. A Fig. 4 mostra imagens obtidas sobre o anteparo para uma amostra de molho shoyu acondicionada num porta-amostra de $200 \mu \mathrm{m}$ de espessura, iluminada com um laser de feixe contínuo (cw) cujo comprimento de onda é $\lambda=532 \mathrm{~nm}$ (Ventus, Laser Quantum) e utilizando uma lente cuja distância focal é $f=10 \mathrm{~cm}$, para diversas potências incidentes.

Como pode ser observado, para baixa potência, não há formação de imagem de difração por automodulação de fase (Fig. 4a). A imagem observada no anteparo é basicamente a mesma que no caso da amostra de água. Aumentando gradualmente a potência do laser há o surgimento de um mínimo na interferência (região escura central na Fig 4b) originando assim o primeiro anel no padrão de interferência. A observação do primeiro anel de interferência acontece quando a diferença de fase entre o centro e a borda do feixe é $2 \pi$. Aumentando gradualmente a potência do laser pode observar-se que o diâmetro do anel de interferência aumenta gradualmente dando lugar a um segundo anel (Fig. 4c). Assim, o aumento da potência incidente sobre a amostra leva tanto a um aumento do diâmetro dos anéis quanto a um número maior de anéis de interferência, alternando-se máximos e mínimos centrais, devido ao aumento na diferença de fase entre o centro do feixe e a borda (4d-f). Em geral, a
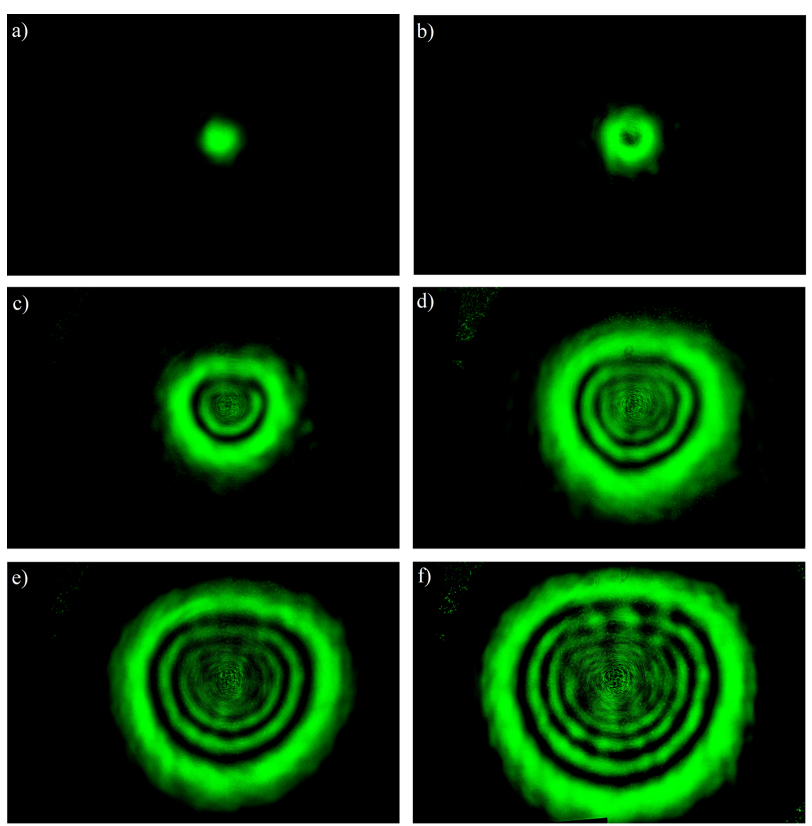

Figura 4: Imagens obtidas sobre um anteparo iluminando uma amostra de molho shoyu num porta-amostra de $200 \mu \mathrm{m}$ utilizando uma lente convergente de $f=10 \mathrm{~cm}$, e com um laser de $\lambda=532 \mathrm{~nm}$ para diversas potências: a) $4 \mathrm{~mW}$, b) $10 \mathrm{~mW}$, c) $26 \mathrm{~mW}$, d) $45 \mathrm{~mW}$, e) $63 \mathrm{~mW}$ e f) $79 \mathrm{~mW}$.

observação de um número $N$ de anéis de interferência $(N$ máximos) corresponderá a uma diferença de fase $N 2 \pi$ entre o centro e a borda do feixe. O padrão de anéis de interferência por automodulação de fase se diferencia do padrão de anéis pela difração de uma abertura circular (disco de Airy) [5, 9] pelo fato que nesta última situação, o aumento da potência do feixe incidente torna evidente um maior número de anéis sem no entanto haver um aumento no diâmetro dos anéis, e existindo sempre um máximo central. Eventualmente e dependendo da intensidade do efeito óptico não linear e do raio do feixe, é possível também observar simultaneamente um padrão de anéis correspondente ao disco de Airy.

O fenômeno de automodulação espacial de fase é devida à existência da absorção óptica do meio, portanto uma diminuição desta implicará na necessidade do uso de um feixe laser de maior potência para a observação do padrão de difração. Como uma forma de confirmar esta afirmação sugere-se diluir gradualmente com água o fluido e comparar o número de anéis observados para uma dada potência com o observado no caso do fluido puro.

\section{Conclusões}

A presente comunicação busca trazer uma contribuição didática para o ensino de tópicos atuais de física, e particularmente óptica, a qual é baseada numa demonstração simples e relativamente econômica de um efeito óptico não linear. Assim, mostramos que é possível abordar de uma maneira simples e sem a necessidade de apelar a todo o arcabouço da matemática para um entendimento 
inicial, um assunto relevante para a formação específica de alunos de graduação da área das ciências básicas e da tecnologia.

\section{Agradecimentos}

Os autores agradecem ao CNPq, Fundação Araucária, Universidade Estadual de Ponta Grossa e Instituto Nacional de Ciência e Tecnologia em Fluidos Complexos (INCT-FCx) pelo apoio financeiro. Um agradecimento especial ao Prof. Dr. Gelson Biscaia de Souza (DeFisUEPG) pela obtenção das imagens.

\section{Referências}

[1] S.C. Zilio, Óptica Moderna-Fundamentos e aplicações (Compacta, São Carlos, 2009).

[2] R.F. Turchiello e S.L. Gómez, Rev. Bras. Ens. Fís. 38, e3501 (2016).

[3] S.R. Lima, G.J. Pereira, D.N. Messias, A.A. Andrade, E. Oliveira, C. Lodeiro, S.C. Zilio, V. Pilla e J. Lumin, Journal of Luminescence 197, 175 (2018).

[4] V. Pilla, E. Munin e M.R.R. Gesualdi, J. Opt. A: Pure Appl. Opt. 11, 105201 (2009).

[5] E. Hecht, Óptica (Calouste Gulbenkian, Lisboa, 2002) $2^{\mathrm{a}}$ ed.

[6] S.L. Gómez, C.S. Lopes, A.A. Bernal-Cardenas, V.M. Lenart e R.F. Turchiello, Rev. Bras. Fis. Tecn. Apl. 3, 18 (2016).

[7] M. Born e E. Wolf, Principles of Optics (Cambridge University Press, Cambridge, 2005).

[8] R. Karimzadeh, Opt. Comm. 286, 329 (2013).

[9] D. Halliday, R. Resnick e J. Walker, Fundamentos de Física (LTC, Rio de Janeiro 2009) $8^{\mathrm{a}}$ ed. 